\title{
The Suggestion of the Drug for COVID-19 with Molecular Docking
}

\author{
reihaneh sabbaghzadeh ${ }^{1}$ \\ ${ }^{1}$ Hakim Sabzevari University
}

June 2, 2020

\begin{abstract}
Coronaviruses (CoV) were reported from Wuhan, China that also transferred from animals to humans. The SARS-CoV-2 spike protein directly binds to the host cell surface ACE2 receptor helping virus entry and replication. The amino acid positions introduced in 2019-nCoV were the corresponding residues in HIV-1 gp120 and HIV-1 Gag. No SARS-CoV-2 therapeutics were available, even if some treatment options have been published which await acceptance. The Autodock software version 1.5.6 and viva were used for the molecular docking process. LIGPLOT software was used for the molecular docking courses. This program automatically generates schematic diagrams of protein-ligand interactions for a given protein in a PDB file (Figure 2). Residues are identical in all seven drugs. The interactions complex between SARS-CoV-2 spike protein complex and seven suggestion drug was done. Binding energies on $\mathrm{kcal} / \mathrm{mol}$, hydrogen bonds, and hydrophobic interactions were calculated. Anakinra is proposed because the only structure that interacts with SARS-CoV-2 spike protein by simulation via software.
\end{abstract}

\section{Introduction}

Coronaviruses (CoV) have single-stranded RNA that infect animals and Humans. Our current understanding is definedas the virus genome sequences and moderate epidemiological and clinical data [1].

In December 2019, the epidemic of coronavirus was reported from Wuhan, China that also transferred from animals to humans [2].

The spike glycoprotein (S) of coronavirus is cut into two subunits ( 1 1 and $\mathrm{S} 2$ ). The $\mathrm{S} 1$ subunit is beneficial in receptor binding and the $\mathrm{S} 2$ subunit helps membrane fusion [3]. Therefore it is desired to investigate the spike glycoprotein of the 2019-nCoV to understand its function, novel features interactions, and structural features using computational tools [1].

In the analysis, the SARS-CoV-2 spike protein directly binds to the host cell surface ACE2 receptor helping virus entry and replication [13].

The 2019- nCoV spike glycoprotein contains 4 insertions. The amino acid positions introduced in 2019-nCoV were the corresponding residues in HIV-1 gp120 and HIV-1 Gag. The first 3 inserts (insert 1, 2 and 3) were aligned to short segments of amino acid residues in HIV-1 gp120, the insert 4 aligned to HIV-1 Gag. The insert 1 ( 6 amino acid residues) and insert 2 (6 amino acid residues) in the spike glycoprotein of $2019-\mathrm{nCoV}$ are $100 \%$ as same as to the residues mapped to HIV-1 gp120. The insert 3 (12 amino acid residues) in 2019-nCoV was corresponded maps to HIV-1 gp120 for gaps, and the insert 4 (8 amino acid residues) maps to HIV-1 Gag for gaps [1].

There are several capability therapeutic approaches, that one of them is the spike protein-based vaccine. Developments of a spike1 subunit protein-based drug that can interact with it maybe have good effect. Because may rely on the fact that ACE2 is the SARS-CoV- 2 receptors [13-14].

Yet, no SARS-CoV-2 therapeutics were available, even if some treatment options which await acceptance have been published, including several broad-spectrum antivirals such as favipiravir and remdesivir and the 
anti-malaria drug chloroquine [10]. It seems that Rheumatoid arthritis (RA), a chronic inflammatory disease that may result in important disability, was connected with COVID-19 [19].

A new hypothesis suggested that angiotensin receptor 1 (AT1R) inhibitors might be beneficial for patients infected by COVID-19 [10]. Then it was guessed that anti-human-interleukin 6 receptors may be a proposal drug with COVIN19. Because the renin-angiotensin system (RAS) is a central mediator in the development of hypertension and associated cardiovascular diseases [9], it was a suggestion to apply AT1R antagonists such as losartan and telmisartan as SARS-CoV-2 therapeutics for treating patients before the development of acute respiratory syndrome remains unproven until tried. At the time of writing this brief commentary, the end of the COVID-19 epidemic is not in sight and forceful actions are required (and being done) for containing its spread and death rate [10].

The drugs were suggested that their effects were approved already on treatment Rheumatoid arthritis (anakinra [20, 22], chloroquine [10]), HIV-1 protease (comostate, lamivudine, pepstatin [13,15]), angiotensinconverting enzyme 2(losartan [6]), antiviral(favipiravir[21]) and cancer (ribavirin [23]) as well as analyzed as therapeutic targets. These targets were objects of interest in different areas of biomedical and pharmaceutical research and the progress and evaluation of bioinformatics, molecular modeling, computer-aided drug design, and analytical tools. Molecular Modeling for the prediction of Selective drugs and their collaborators were focused on the selection of them since the last decade, with the help of molecular modeling methods in some instances.

Development of advanced computational methods for bioinformatics, molecular modelingand drug design, introduces known therapeutic targets to refine and use algoritms.

\section{Materials and Methods}

Drugs were prepared using Chem Draw 8.0. The $5 \mathrm{wrg}$ molecular model (from the PDB) was used in the simulated docking studies for SARS-CoV-2 spike protein as receptor protein. Input protein structures were prepared by adding hydrogen atoms and removing non-functional water molecules. The Autodock software version 1.5.6 (Table 1) and viva (Figure 1) were used for the molecular docking process. The Lamarckian Genetic Algorithm method was used for the global optimum binding position search. The torsion angles of the drugs were identified, hydrogens were added to the macromolecule, bond distances were edited and solvent parameters were added to the enzyme 3D structure. Partial charges were calculated using Gasteiger's method. Docking parameters were as follows: population size of 150, the maximum number of energy evaluation ranges of 25.0000 , the maximum number of generations is 27,000 , the mutation rate of 0.02 , cross-over rate of 0.8 . Other docking parameters were set to the software at its default values. After docking, the drugs were ranked according to their docked energy as implemented in the AutoDock program. A residue ASN 479 in protein binding site was also chosen due to its possible specific hydrogen bonds. This residue was set as flexible residue, while the other residues were kept as rigid residues.

Several 100 cycles of calculation were used to get a final binding position as accurately as possible. The docking procedure was run and the maximum negative Final Docking Energy was calculated. The following parameters were set during docking simulation: population size, 150; and max steps, 100. The center of a grid size were with 40,40,40, and 221, 185, 118 points in $\mathrm{X}, \mathrm{Y}$, and $\mathrm{Z}$ axes respectively. Both were with a grid spacing of $0.0375 \mathrm{~nm}$ and were centered at the experimentally determined binding sites.

LIGPLOT software was used for the molecular docking courses. This program automatically generates schematic diagrams of protein-ligand interactions for a given protein in a PDB file (Figure 2). Residues are identical in all seven drugs. The SARS-CoV-2 spike protein structure was constituted of strong hydrogen bonds and hydrophobic interactions, that was observed involving in the protein: drug structure.

\section{Results}

Docking studies conclude the interaction of drugs with protein and residues involved in this complex. For such interaction studies, the most important condition was the proper orientation and conformation of drugs which fitted to the protein binding site appropriately and formed the protein-drug complex. Therefore, 
optimal interactions and docking results were defined. Docking and MD simulations were used in the molecular modeling protocol which accepted that the ASN479 group could have favorable interactions in the S1 subunit of SARS-CoV-2 spike protein.

Molecular modeling found the orientation with plots that were shown in Figure 1, where the ASN residue 479 was placed to interact with drugs. In this position, the asparagine of the SARS-CoV-2 spike protein is close to the residues that were connected by hydrophobic interactions and hydrogen bonds. In Anakinra drug, TYR442, TRP476, LEU478, LYS439, and ASP480 were contributed in addition to ASN479 by hydrophobic interactions (figure 2-a). In chloroquine drug, TYR440, ASP480, LEU478, and TYR442 were contributed in addition to ASN479 by hydrophobic interactions (Figure 2-b). In comostat drug, LEU478, ASP480, and TYR440 were contributed in addition to ASN479 by hydrophobic interactions (Figure 2-c). In favipiravir drug, TYR442, TYR440, and LEU478 were contributed in addition to ASN479 by hydrophobic interactions (Figure 2-d). In lamivudine drug, TYR442, TYR440, and ASP480 were contributed in addition to ASN479 by hydrophobic interactions (Figure 2-e). In losartan drug, TYR440, ASP480, LEU478, and TYR442 were contributed in addition to ASN479 by hydrophobic interactions (Figure 2-f). In the ribavirin drug, TYR440, ASP480, LEU478, and TYR442 were contributed in addition to ASN479 by hydrophobic interactions (Figure 2-h). Also it was shown with LigPlot software that drugs (b, c, d, e, f, and h) have hydrogen bonds with the protein in addition to. However, Orientations of this residues with drugs were approved by autodock vina (figure 1).

Table1: Binding energy between SARS-CoV-2 spike protein and drugs.

There are many similarities of SARS-CoV-2 with the original SARS-CoV. Using computer modeling it was found that the spike proteins of SARS-CoV-2 and SARS-CoV have almost identical 3-D structures in the receptor-binding domain that maintains van der Waals forces [13]. Therefore interactions of hydrophobic were concluded to treatment.

Fig 1. The plots generated by autodock vina software. These interactions are complex between SARS-CoV2 spike protein complex; a) anakinra (binding energy=-7.4 kcal $/ \mathrm{mol}$ ), b) chloroquine (binding energy=$7.2 \mathrm{kcal} / \mathrm{mol}$ ), c) comostat (binding energy=-8.8 kcal $/ \mathrm{mol}$ ), d) favipiravir (binding energy=-5 kcal $/ \mathrm{mol}$ ), e) lamirudine (binding energy $=-6 \mathrm{kcal} / \mathrm{mol}$ ), f) lostartan (binding energy=-6.7 kcal $/ \mathrm{mol}$ ), g) pepstatin (binding energy $=-9.5 \mathrm{kcal} / \mathrm{mol}$ ), h) ribavirin (binding energy $=-6.9 \mathrm{kcal} / \mathrm{mol}$ ).

Fig 2. The plots generated by LigPlot ${ }^{+}$program. Hydrogen binding (blue line) and hydrophobic interactions ( red line) pockets of SARS-CoV-2 spike protein: drugs complexes; a) anakinra, b) chloroquine, c) comostat, d) favipiravir, e) lamivudine, f) losartan and h) ribavirin.

\section{Discussion}

The physiological role of Interleukin 6 (IL-6) has been advised to participation in the pathogenesis of several diseases. These introduce multiple myeloma, post-menopausal osteoporosis, and chronic autoimmune diseases, where a stable association has been shown between disease changes and increased production of the cytokine. For these reasons it is commonly believed that potent IL-6 receptor antagonists will be helpful therapeutic tools [4].

On the other hand, the renin-angiotensin system (RAS), a peptide based system, has been classically recognized as a complex linear humoral system. It gives to the control of cardiovascular, renal, and adrenal functions [1]. The circulatory protease, renin is a key enzyme of the RAS and is secreted. The RAS is one of the central members in respiratory diseases such as acute respiratory distress syndrome (ARDS) and acute lung injury (ALI) during sepsis [5]. ARDS is characterized by pulmonary edema, severe hypoxia, and accumulation of inflammatory cells [10]. One of the common indications of ARDS is a strong inflammatory response, which is characterized by the release of proinflammatory cytokines [5].

The effect of RAS is well known in the cardiovascular system in specific in the control of blood pressure, vasoconstriction, cell growth and cardiac remodelling. Inflammation and oxidative stress play an important role with it $[6]$. 
Also, Rheumatoid arthritis (RA) is an inflammatory disease of joints. It is related with increased risk of cardiovascular complications. The main disadvantageous contributing factor for an increased mortality in RA is the extent of associated systemic inflammation. Then it is opinion that drugs was guessed can be effective and is suggested that pepstatin inhibitor can be appropriate on interaction with SARS-CoV-2 for future work.

Two models of chemical series selected from the above mentioned database were modified these compounds by including substituents to have interactions with SARS-CoV-2 spike protein receptor residues at S1 subunit.

The purpose of this article was to brief about antiSARS-CoV-2 spike protein drugs (Pharmacokinetics and pharmacodynamics). Activated inflammation, as demonstrated by persistently higher IL-6 levels, may have profound and far-reaching clinical implications [16]. Even when virologically contained, treated HIVinfected persons have significantly higher plasma levels of IL-6 than well-matched uninfected controls [17]. Changes in cytokine levels in HIV infected individuals can affect the function of the immune system and have the potential to directly impact the course of HIV disease effects of cytokines are pleiotropic and are influenced by the concentrations, presence or absence of other cytokines. Once produced, these factors may act individually or together, directly or indirectly on infected cells, activating cellular components of the intrinsic system and/or promoting specific T- and B-cell adaptive responses to mediate anti-microbial effects [18].

There are a number of pharmaceuticals before being tested, but a better understanding of the underlying pathobiology is required. In this context, this article was briefly reviewed the hypothesis for SARS-CoV-2 spike protein receptor as a specific target.

The same protocol applied to drugs forming complexes with ASN479. Analysis of the number of hydrogen and hydrophobic bonds between drugs and protein shows that the complex has a higher number of intermolecular Van der Waals bonds, which indicates that it has higher affinity for TYR442, TRP476, LEU478, LYS439 and ASP480 than others.

Theoretically, lostartan was the lowest energy obtained in docking simulations, so that should be the best selection, but comparison RefRMSs to expression standard deviation and the consideration inhibitor constant, lamirudine can be the most effective and then favipiravir as well as in the end, chloroquine, respectively. Hawever, anakinra is proposed, because the only structure that interact with SARS-CoV-2 spike protein by simulation vina software (Figure1-a).

\section{References}

[1] Prashant Pradhan, Ashutosh Kumar Pandey, Akhilesh Mishra, Parul Gupta1, Praveen Kumar Tripathi1, Manoj Balakrishnan Menon1, James Gomes1, Perumal Vivekanandan and Bishwajit Kundu, Uncanny similarity of unique inserts in the 2019-nCoV spike protein to HIV-1 gp120 and Gag, bioRxiv, doi: https://doi.org/10.1101/2020.01.30.927871.

[2] Jasper Fuk-Woo Chan, Kin-Hang Kok, Zheng Zhu, Hin Chu, Kelvin Kai-Wang To, Shuofeng Yuan \& Kwok-Yung Yuen, Genomic characterization of the 2019 novel human pathogenic coronavirus isolated from a patient with atypical pneumonia after visiting Wuhan, Emerging Microbes \& Infections, 2020.

[3] Berend Jan Bosch, Ruurd van der Zee, Cornelis A. M. de Haan, and Peter J. M. Rottier, The Coronavirus Spike Protein Is a Class I Virus Fusion Protein: Structural and Functional Characterization of the Fusion Core Complex, JOURNAL OF VIROLOGY, Aug. 2003, p. 8801-8811 Vol. 77, No. 16.

[4] R.Savino, LCiapponi, A.Lahm1, A.Demartis, A.Cabibbo, C.Toniatti, PRDelmastro2, S.Altamura and G.Ciliberto3, Rational design of a receptor super-antagonist of human interleukin-6, The EMBO Journal vol.13 no. 24 pp.5863-5870, 1994.

[5] Ravinder Reddy Gaddam, Stephen Chambers and Madhav Bhatia, ACE and ACE2 in Inflammation: A Tale of Two Enzymes, Inflammation \& Allergy - Drug Targets, 2014. 
[6] Hai-Yan Jin, Bei Song, Gavin Y. Oudit, Sandra T. Davidge, Hui-Min Yu, Yan-Yan Jiang,

Ping-Jin Gao1,2, Ding-Liang Zhu1,2, Guang Ning1, Zamaneh Kassiri3, Josef M. Penninger6, Jiu- Chang Zhong, ACE2 Deficiency Enhances Angiotensin II-Mediated Aortic Profilin-1 Expression, Inflammation and Peroxynitrite Production, PLoS ONE, June 2012, Volume 7, Issue 6.

[7] Ruiz-Ortega M, Esteban V, Rupérez M, Sánchez-López E, Rodríguez-Vita J, Carvajal G, Egido J, Renal and vascular hypertension-induced inflammation: role of angiotensin II. , Curr Opin Nephrol Hypertens. 2006 Mar;15(2):159-66.

[8] Tianxi Cai, ScD; Yichi Zhang, PhD; Yuk-Lam Ho, MPH; Nicholas Link, BA; Jiehuan Sun, PhD; Jie Huang, MS; Tianrun A. Cai, MD; Scott Damrauer, MD; Association of Interleukin 6 Receptor Variant With Cardiovascular Disease Effects of Interleukin 6 Receptor Blocking Therapy A Phenome-Wide Association Study, JAMACardiology.

[9] Mohammed A.R. Chamsi-Pasha, MD1, Zhili Shao, MD PhD2, and W. H. Wilson Tang, MD2, AngiotensinConverting Enzyme 2 as a Therapeutic Target for Heart Failure, Curr Heart Fail Rep, 2015.

[10] David Gurwitz, Angiotensin receptor blockers as tentative SARS-CoV-2 therapeutics,2020, https://doi.org/10.1002/ddr.21656.

[11] Julio Caballero, Considerations for Docking of Selective Angiotensin-Converting Enzyme Inhibitors, molecules, 2020, 25, 295.

[12] Lanying Du, Yuxian He, Yusen Zhou, Shuwen Liu, Bo-Jian Zheng[?], and Shibo Jiang, The spike protein of SARS-CoV - a target for vaccine and therapeutic development, Nat Rev Microbiol. 2009 March ; 7(3): 226-236.

[13] Haibo Zhang, Josef M. Penninger, Yimin Li, Nanshan Zhong and Arthur S. Slutsky, Angiotensinconverting enzyme 2 (ACE2) as a SARS-CoV-2 receptor: molecular mechanisms and potential therapeutic target, Intensive Care Med, 2020.

[14]Yun Chen et al., Biochemical and Biophysical Research Communications, 2020.

[15] Vaidya KA, Kadam AV and Nema V, Anti-Retroviral Drugs for HIV: Old and New, Austin Journal of HIV/AIDS Research, 2016.

[16] Alvaro H. Borges,1 Jemma L. O'Connor,2 Andrew N. Phillips,2 Frederikke F. Ronsholt,3 Sarah Pett,2,4,5 Michael J. Vjecha,6 Martyn A. French,7,8 and Jens D. Lundgren1; for the INSIGHT SMART and ESPRIT Study Groups and the SILCAAT Scientific Committee, Factors Associated With Plasma IL-6 Levels During HIV Infection, JID 2015:212.

[17] Faustina Nkechi Osuji1, Charles Chinedu Onyenekwe, Joseph Ebere Ahaneku and Nkiruka Rose Ukibe, The effects of highly active antiretroviral therapy on the serum levels of proinflammatory and antiinflammatory cytokines in HIV infected subjects, Osuji et al. Journal of Biomedical Science (2018) 25:88.

[18] Christine Rogez-Kreuz1, Benjamin Mane' glier1, Marc Martin1, Nathalie Dereuddre-Bosquet, Jacques Martal, Dominique Dormont and Pascal Clayette, Involvement of IL-6 in the anti-human immunodeficiency virus activity of IFN-s in human macrophages, International Immunology, Vol. 17, No. 8, pp. 1047-1057.

[19] By Rachel A. Burke, Pharm.D., BCACP; and Nicole D. White, Pharm.D., Biologic Disease-Modifying Antirheumatic Drugs, PSAP 2014.

[20] H. Haibel and C. Specker, Disease-modifying anti-rheumatic drugs in rheumatoid arthritis and ankylosing spondylitis, CLINICAL AND EXPERIMENTAL RHEUMATOLOGY 2009.

[21] Yousuke Furuta et al., T-705 ( favipiravir) and related compounds, Antiviral Research, 82(2009) 95-102.

[22] Jasvinder A Singh, Alomgir Hossain, Elizabeth Tanjong Ghogomu, Ahmed Kotb, Robin ChristensenAmy S Mudano, Lara J Maxwell, Nipam P Shah, Peter Tugwell, George A Wells, Biolog- 
ics or tofacitinib for rheumatoid arthritis in incomplete responders to methotrexate or other traditional disease-modifying anti-rheumatic drugs: a systematic review and network meta-analysis, https://doi.org/10.1002/14651858.CD012183.

[23] WHO Model Formulary 2008.

\section{Hosted file}

Fig .docx available at https://authorea.com/users/328948/articles/456063-the-suggestion-ofthe-drug-for-covid-19-with-molecular-docking

\section{Hosted file}

Table1.docx available at https://authorea.com/users/328948/articles/456063-the-suggestionof-the-drug-for-covid-19-with-molecular-docking 